\title{
Omentum-preserving gastrectomy for advanced gastric cancer: a propensity-matched retrospective cohort study
}

\author{
Shinichi Hasegawa $\cdot$ Chikara Kunisaki $\cdot$ Hidetaka Ono $\cdot$ Takashi Oshima \\ Shoichi Fujii $\cdot$ Masataka Taguri $\cdot$ Satoshi Morita $\cdot$ Tsutomu Sato $\cdot$ \\ Roppei Yamada $\cdot$ Norio Yukawa $\cdot$ Yasushi Rino $\cdot$ Munetaka Masuda
}

Received: 9 May 2012/Accepted: 4 September 2012/Published online: 17 September 2012

(c) The International Gastric Cancer Association and The Japanese Gastric Cancer Association 2012

\begin{abstract}
Background and objectives We clarified the impact of omentectomy for advanced gastric cancer on patient survival from the surgical results of a high-volume center in Japan.

Methods Patients who received curative gastrectomy were divided into two groups based on whether they underwent omentectomy. The propensity score-matching method was used to assemble a well-balanced cohort, and relapse-free survival and the pattern of recurrence were compared.

Results For this study, 330 patients who fulfilled the inclusion criteria participated and were divided into two groups: group $\mathrm{R}$, patients who received omentectomy, and group $P$, patients who received omentum-preserving gastrectomy. After performing score-matching, 196 patients were selected. The 3- and 5-year relapse-free survival rates were $72.9 \%$ (95\% confidence interval, 64.1-81.7) and $66.2 \%(56.6-75.8 \%)$ in group R, and $76.7 \%(67.9-81.2)$ and $67.3 \%(55.1-79.5)$ in group $\mathrm{P}$, which were not significantly different $(P=0.750)$. Regarding sites of relapses, no differences were observed between the groups $(P=0.863)$.
\end{abstract}

S. Hasegawa $(\bowtie) \cdot$ C. Kunisaki · H. Ono - T. Oshima · S. Fujii Department of Surgery, Gastroenterological Center,

Yokohama City University Medical Center, Urafune-cho 4-57,

Minami-ku, Yokohama 232-0024, Japan

e-mail: hsgws@yokohama-cu.ac.jp

M. Taguri · S. Morita

Department of Biostatistics and Epidemiology,

Yokohama City University, Yokohama, Japan

T. Sato $\cdot$ R. Yamada $\cdot$ N. Yukawa $\cdot$ Y. Rino $\cdot$ M. Masuda Department of Surgery, Yokohama City

University, Yokohama, Japan
Conclusions In this series, omentum-preserving gastrectomy for advanced gastric cancer did not increase the peritoneal relapse rate or affect patient survival compared to conventional gastrectomy. The non-inferiority of the omission of omentectomy should be evaluated by a randomized controlled trial.

Keywords Gastric cancer - Surgery · Omentum · Omentectomy

\section{Introduction}

The omentum is a mesenteric tissue apron that hangs down from the stomach, providing a protective cushion and reduction of intestinal adhesion. It also serves as an antibacterial defense, absorbs foreign materials in the abdominal cavity, and provides a defense against an inflammatory site [1, 2]. A kind of lymphoid tissue, the so-called milky spot, exists in the omentum and acts as a gate through the abdominal cavity into the subperitoneum [3], which contributes to the peritoneal seeding of cancer cells $[4,5]$. Thus, complete resection of the greater omentum has been believed to be essential to eliminate tumor cells during surgery for gastric cancer $[6,7]$.

However, there has so far been no evidence showing a definitive improvement of survival after gastrectomy with omentectomy, even for cases of advanced gastric cancer. In the Japanese gastric cancer treatment guidelines 2010 (version 3) edited by the Japanese Gastric Cancer Association, there is no definite comment regarding omentectomy; instead, the guidelines state that 'Removal of the greater omentum is usually integrated in the standard gastrectomy for T3 (SS) or deeper tumors. For T1/2 
tumors, the omentum more than $3 \mathrm{~cm}$ away from the gastroepiploic arcade may be preserved [8].' The complete removal of the peritoneum from the abdominal cavity is theoretically impossible; therefore, the effect of omentectomy on the prevention of peritoneal relapse may be limited. Moreover, adjuvant S-1 monotherapy after curative gastrectomy for stage II/III gastric cancer has been established on the basis of a phase III trial conducted in Japan [9], which indicated that systemic chemotherapy may eliminate micrometastatic disease better than surgical intervention. In fact, some studies reported from Japan demonstrated that omentum-preserving gastrectomy for advanced gastric cancer might not have any significant effect on patient survival [10, 11]. Recently, laparoscopic surgery for gastric cancer has become widespread in Japan, and several clinical trials of laparoscopic gastric surgery for advanced disease are now ongoing. The greater omentum is usually preserved in laparoscopic surgery.

We herein attempted to evaluate the impact of omentectomy for advanced gastric cancer on patient survival based on the surgical results of a high-volume center in Japan.

\section{Materials and methods}

\section{Study population}

Patients who underwent curative gastrectomy for advanced gastric cancer were selected from a prospective database established at the Gastroenterological Center, Yokohama City University Medical Center. The inclusion criteria were (1) histologically proven adenocarcinoma, (2) having undergone a gastrectomy, (3) pT2-4, N0-3, M0, (4) received D1 or more radical lymphadenectomy, and (5) achieved R0 resection. Patients with type 4 tumors were excluded because their pathogenesis may have been different from that of other patients. Patients with positive peritoneal lavage were also excluded. Those who fulfilled the criteria were divided into two groups: group R, patients who received conventional gastrectomy with a complete resection of the omentum; and group $\mathrm{P}$, patients who received omentum-preserving gastrectomy. We used propensity score-matching to assemble a cohort in which patients who received omentum-resected and omentumpreserving gastrectomy would be balanced on the basis of their baseline characteristics. Each patient in group $\mathrm{P}$ was matched by age, gender, p-stage, and the extent of lymph node dissection to a patient in group R. After adjusting such factors, relapse-free survival and the pattern of recurrence were compared between the matched cohorts. Overall survival and postoperative complications, including ileus, were also evaluated.
Surgical procedures

Omentum-preserving gastrectomy was performed as follows: the greater omentum was dissected $3 \mathrm{~cm}$ away from the gastroepiploic arcade, and the greater omentum on the transverse colon side was preserved. Following this procedure, distal or total gastrectomy with D2 dissection was performed. The reconstruction was performed by the Roux-en-Y method in patients undergoing total gastrectomy, and by the Billroth-I or Roux-en-Y method in those undergoing distal gastrectomy, with an end-to-side anastomosis using a circular stapler. On the other hand, when a conventional gastrectomy was performed, the transverse colon and the greater omentum were divided at the thin membrane beside the colon, and the omentum was completely resected. We have performed omentum-preserving gastrectomy for advanced gastric cancer prospectively since 2007 on the basis of a previous Japanese study $[10,11]$ when patients provided their acceptance. The institutional review board approved this study.

\section{Clinical evaluation}

The UICC TNM classification (7th edition) was used to evaluate tumor progression, histopathological grading, and residual tumor (R) classification. Postoperative complications were diagnosed based on Clavien's system [12].

Statistical analysis

All data were presented as the median and total range unless otherwise stated. The chi-square test was used to compare proportions. Student's $t$ test was performed to compare continuous data. Survival was calculated by the Kaplan-Meier method and was then compared by the logrank test. Cox's proportional hazard model was also used to perform the survival analysis. $P<0.05$ was considered statistically significant.

\section{Results}

Baseline characteristics

Between January 2000 and December 2009, 330 patients fulfilled the inclusion criteria and participated in this study. The patients, 231 men and 99 women, had a mean age of 67.9 years (range, 39-91). A change in the proportion of each group is shown in Fig. 1.

The baseline characteristics of the study population are shown in Table 1 . The patients in group $\mathrm{R}$ were younger, had more progressive disease, and received more radical surgical treatment than those in group P. In addition, a 
Fig. 1 Change in proportions of each group

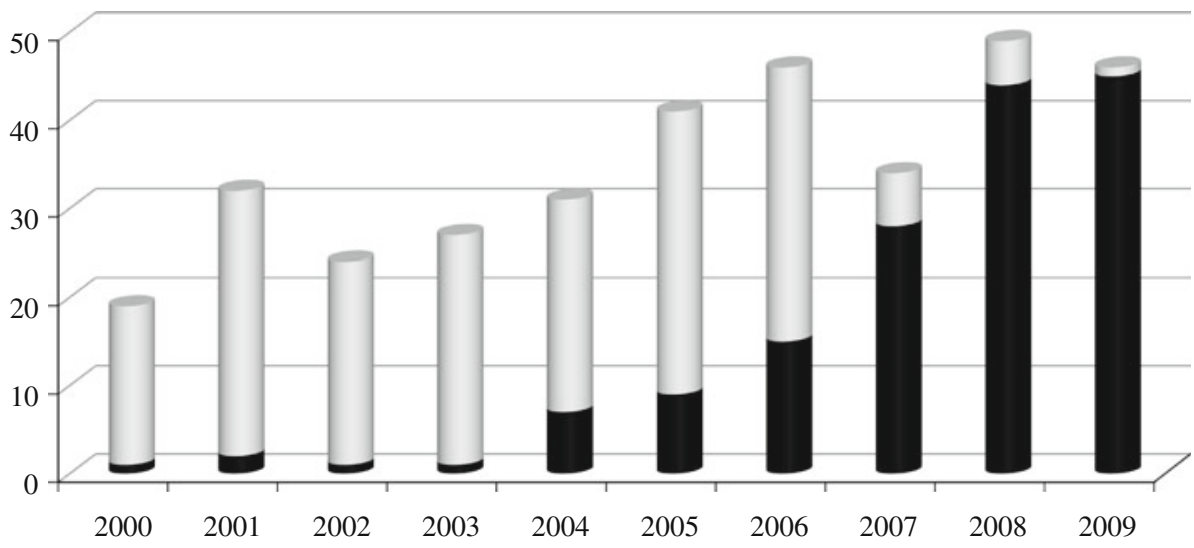

difference was observed in the period in which they underwent gastrectomy. As demonstrated in Table 1, the baseline characteristics were well balanced in the matched populations, although the surgical procedures, the period in which patients received gastrectomy, and the proportion of the patients who received adjuvant chemotherapy differed between the groups. No statistical differences in either relapse-free survival or overall survival were observed between the matched patients and those excluded $(P=0.915$ and 0.563 , respectively).

\section{Surgical outcomes}

Figure 2 shows the relapse-free survival curves for the two groups. The median follow-up time of the survivors in each group was 5.1 (range, 0.5-10.9) years in group $\mathrm{R}$ and 3.3 (range, 0.3-5.8) years in group P, respectively. The 3- and 5-year relapse-free survival rates were $72.9 \% \quad(95 \%$ confidence interval, 64.1-81.7) and 66.2\% (56.6-75.8\%) in group $\mathrm{R}$, and $76.7 \%(67.9-81.2)$ and $67.3 \%$ (55.1-79.5) in group $\mathrm{P}$, which were not significantly different ( $P=0.750$, by the log-rank test). When stratified by histopathological type, relapse-free survival did not differ substantially between the groups $(P=0.668$ in differentiated type; $P=0.978$ in undifferentiated type). The sites of relapse in each group are shown in Table 2; no significant difference was observed between the groups $(P=0.863)$. Figure 3 shows relapse-free survival curves limited to the patients with pT3/T4 tumors (Fig. 3a) and to those with pT4 tumors (Fig. 3b). No significant differences were observed between the two groups $(P=0.668$ and $P=0.949$, respectively).

Overall survival was not statistically different between the groups, as shown in Fig. 4. The 3- and 5-year survival rates were $77.9 \%(69.5-86.3)$ and $66.6 \%(57.0-76.2)$ in group $\mathrm{R}$ and $89.3 \%(82.6-96.0)$ and $79.6 \%(68.2-91.0)$ in group $\mathrm{P}(P=0.051)$. A univariate Cox analysis for overall survival demonstrated no statistical difference in the overall survival between the two groups $(P=0.055$, Table 3). Elapsed time of the operation was longer and the amount of bleeding was significantly higher in group $\mathrm{R}$ than in group $\mathrm{P}$, whereas the number of patients who suffered postoperative complications and ileus did not differ, as demonstrated in Table 4.

\section{Discussion}

In Japan, gastric cancer is the most common malignancy and one of the leading causes of cancer death. Peritoneal dissemination is the most common type of recurrence after curative gastrectomy. Therefore, the omentum is usually resected to eliminate the microscopic seeds on the assumption that the omentum facilitates the spread of malignancy. However, few studies have focused on the effect of omentectomy on the patient survival. Fujita et al. [10] compared the surgical results of omentum-preserving gastrectomy for advanced gastric cancer (pT2-T4a) to those for conventional gastrectomy. They demonstrated that long-term results did not differ between the groups, whereas the incidence of postoperative complications was higher in the patients who received conventional gastrectomy. Watanabe et al. [11] also reported similar results. Those studies suggested that omentum-preserving gastrectomy might be beneficial for patients with advanced gastric cancer. However, both reports had problems with comparability between the groups; namely, the patients who received omentectomy had more progressive disease than those received omentum-preserving surgery. 
Table 1 Baseline characteristics and propensity score-matched baseline characteristics between group R (patients who underwent omentectomy) and group $\mathrm{P}$ (patients who underwent omentum-preserving gastrectomy)

\begin{tabular}{|c|c|c|c|c|c|c|}
\hline & \multicolumn{3}{|l|}{ Baseline } & \multicolumn{3}{|c|}{ Propensity score-matched baseline } \\
\hline & Group $\mathrm{R}(N=180)$ & Group $\mathrm{P}(N=150)$ & $P$ value & Group $\mathrm{R}(N=98)$ & Group $\mathrm{P}(N=98)$ & $P$ value \\
\hline Mean age (range) & $66.7(39-91)$ & $69.4(44-91)$ & 0.01 & $69.0(40-91)$ & $68.7(45-91)$ & 0.813 \\
\hline Gender (male/female) & $126 / 54$ & $105 / 45$ & 1.000 & $72 / 26$ & $72 / 26$ & 1.000 \\
\hline Comorbidity & 100 & 98 & 0.071 & 51 & 61 & 0.149 \\
\hline \multicolumn{7}{|l|}{ Period } \\
\hline $2000-2005$ & 142 & 20 & \multirow[t]{2}{*}{$<0.001$} & 79 & 14 & \multirow[t]{2}{*}{$<0.001$} \\
\hline 2006-2009 & 32 & 130 & & 19 & 84 & \\
\hline \multicolumn{7}{|l|}{ pT } \\
\hline $\mathrm{pT} 2$ & 43 & 55 & \multirow[t]{4}{*}{0.047} & 30 & 34 & \multirow[t]{4}{*}{0.779} \\
\hline pT3 & 75 & 50 & & 34 & 30 & \\
\hline pT4a & 60 & 45 & & 34 & 34 & \\
\hline pT4b & 2 & 0 & & 0 & 0 & \\
\hline \multicolumn{7}{|l|}{$\mathrm{pN}$} \\
\hline pNO & 66 & 66 & \multirow[t]{4}{*}{0.004} & 39 & 41 & \multirow[t]{4}{*}{0.979} \\
\hline pN1 & 46 & 29 & & 25 & 23 & \\
\hline $\mathrm{pN} 2$ & 23 & 36 & & 18 & 17 & \\
\hline $\mathrm{pN} 3$ & 43 & 19 & & 16 & 17 & \\
\hline \multicolumn{7}{|l|}{ p-stage } \\
\hline Stage I & 23 & 34 & \multirow[t]{4}{*}{0.06} & 16 & 21 & \multirow[t]{4}{*}{0.638} \\
\hline Stage II & 80 & 58 & & 40 & 36 & \\
\hline Stage III & 77 & 58 & & 42 & 41 & \\
\hline Stage IV & 0 & 0 & & 0 & 0 & \\
\hline \multicolumn{7}{|l|}{ Histopathological type } \\
\hline Differentiated & 109 & 82 & \multirow[t]{2}{*}{0.281} & 61 & 58 & \multirow[t]{2}{*}{0.661} \\
\hline Undifferentiated & 71 & 68 & & 37 & 40 & \\
\hline \multicolumn{7}{|l|}{ Approach } \\
\hline Open gastrectomy & 180 & 110 & \multirow[t]{2}{*}{$<0.001$} & 98 & 84 & \multirow[t]{2}{*}{$<0.001$} \\
\hline Laparoscopic gastrectomy & 0 & 40 & & 0 & 14 & \\
\hline \multicolumn{7}{|l|}{ Surgical procedure } \\
\hline Total gastrectomy & 93 & 57 & \multirow[t]{2}{*}{0.013} & 46 & 37 & \multirow[t]{2}{*}{0.193} \\
\hline Distal gastrectomy & 87 & 93 & & 52 & 61 & \\
\hline Total bursectomy & 113 & 0 & $<0.001$ & 58 & 0 & $<0.001$ \\
\hline Splenectomy & 59 & 11 & $<0.001$ & 26 & 10 & 0.003 \\
\hline \multicolumn{7}{|c|}{ Extent of lymph node (LN) dissection } \\
\hline $\mathrm{D} 1+\alpha$ & 10 & 10 & \multirow[t]{4}{*}{$<0.001$} & 9 & 10 & 0.996 \\
\hline $\mathrm{D} 1+\beta$ & 3 & 45 & & 3 & 3 & \\
\hline D2 & 134 & 93 & & 84 & 83 & \\
\hline $\mathrm{D} 2+$ & 33 & 2 & & 2 & 2 & \\
\hline Neoadjuvant chemotherapy & 6 & 4 & 0.702 & 4 & 2 & 0.407 \\
\hline Adjuvant chemotherapy & 38 & 48 & 0.025 & 20 & 34 & 0.025 \\
\hline
\end{tabular}

Our results support their conclusions. Relapse-free survival and the sites of relapse did not differ between the two matched cohorts, suggesting that omentum-preserving gastrectomy for advanced gastric cancer may not increase the risk of peritoneal relapse. Overall survival was not statistically different between the two groups, although it was slightly better in group P; this may be explained by differences in the systemic chemotherapy that the patients received after recurrence. Even in the matched cohort, the period in which patients received gastrectomy was different between the groups (Table 1), and the median follow-up times were shorter in group $\mathrm{P}$, which may have affected these results. 


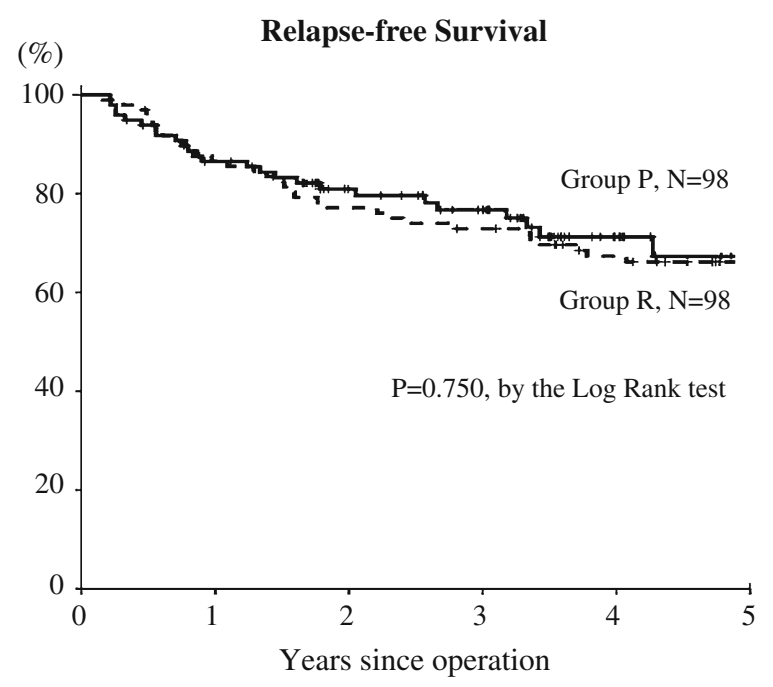

No. at Risk

Group R 98

Group P 98

83
81

73
61

68

50

58

23

46

Fig. 2 Relapse-free survival in all matched patients stratified by treatment group

Table 2 Initial site of relapse in each group

\begin{tabular}{lll}
\hline & Group R $(n=98)$ & Group P $(n=98)$ \\
\hline Peritoneum & 7 & 7 \\
Liver & 7 & 8 \\
Lymph node & 3 & 2 \\
Distant organ & 1 & 3 \\
\hline
\end{tabular}

$P=0.863$

Regarding postoperative complications, Ha et al. [13] reported the effects of omentum-preserving gastrectomy on early and late abdominal complications. They demonstrated that the rate of abdominal complications was lower in the omentum-preserving group compared to the omentumresected group $(P=0.026)$. Fujita et al. and Watanabe et al. $[10,11]$ reported similar results. In this study, the rate of postoperative complications was slightly lower in group $\mathrm{P}$ (37.8\% vs. $40.8 \%$ ), but the difference did not reach statistical significance. The duration of the operation was longer, and the amount of bleeding was greater, in group R, which may imply that the patients in group $\mathrm{R}$ underwent more aggressive surgery than those in group $\mathrm{P}$. More than half of the patients in group $\mathrm{R}$ received total bursectomy as well as omentectomy, and the number of the patients who received splenectomy was greater in group $\mathrm{R}(P=0.003)$. These differences in each surgical procedure, other than the omentectomy itself, may have concealed the effects of omentectomy on surgical morbidity.

Recently, a randomized controlled trial to evaluate the impact of omentobursectomy was reported from Japan, which indicated that this procedure might improve patient a Relapse-free Survival of the patients with pT3/4 tumors

$$
\text { (\%) }
$$

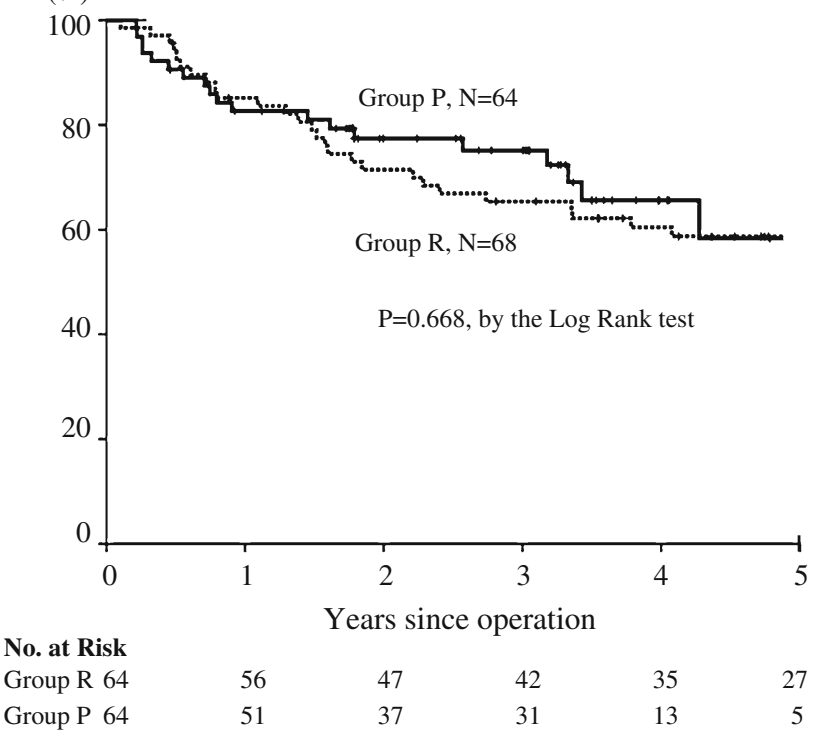

b Relapse-free Survival of the patients with pT4 tumors

(\%)

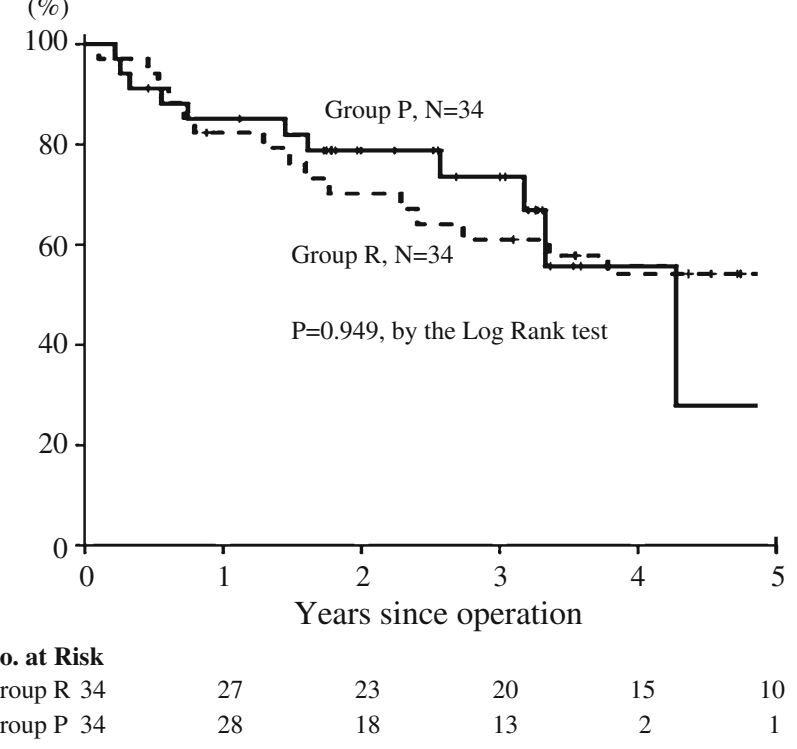

Fig. 3 Relapse-free survival limited to matched patients with pT3 and pT4 tumors (a) and those with pT4 tumors (b)

survival [14]. Although the trial was slightly underpowered, this is thought to be the first report that evaluated the impact of bursectomy on survival. A phase III study is ongoing in Japan to confirm these results.

Controversy still exists about the contribution of surgical intervention to the elimination of cancer cells for the prevention of peritoneal relapse. Although we tried to minimize any selection bias, our study was a retrospective analysis in which various therapeutic factors, e.g., total bursectomy, splenectomy, and adjuvant chemotherapy, may also have affected the results. 


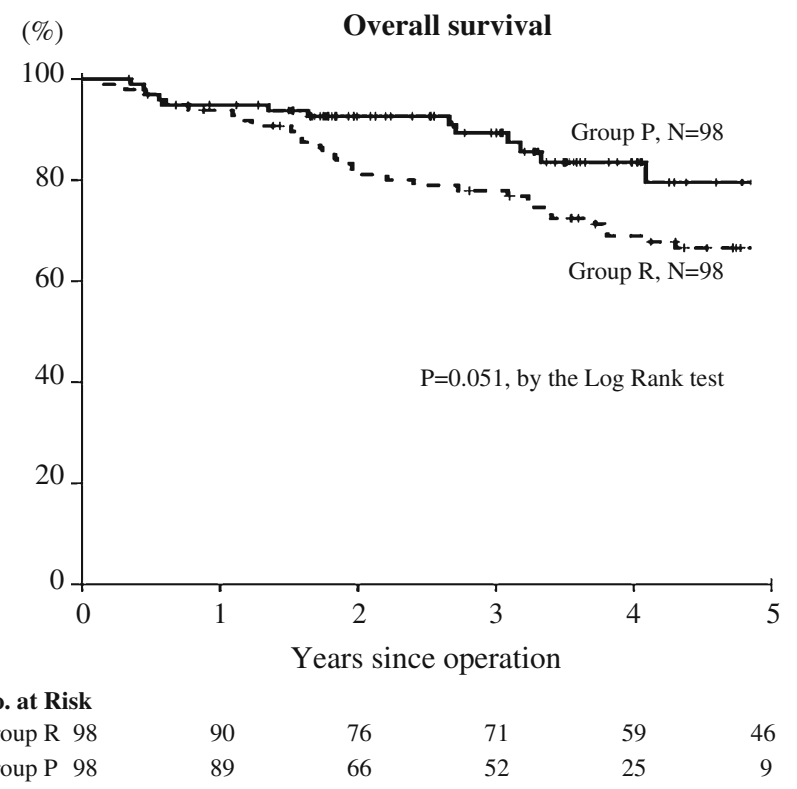

Fig. 4 Overall survival in all matched patients stratified by treatment group

Table 3 Univariate Cox proportional hazards analysis of omentectomy

\begin{tabular}{lllll}
\hline & No. of patients & HR & $95 \%$ CI & $P$ value \\
\hline Group R & 98 & 1.000 & - & 0.055 \\
Group P & 98 & 0.527 & $0.274-1.012$ & \\
\hline
\end{tabular}

$H R$ hazard ratio, $\mathrm{CI}$ confidence interval

Table 4 Comparison of length of operation, amount of bleeding, and postoperative morbidity

\begin{tabular}{lcrr}
\hline & $\begin{array}{l}\text { Group R } \\
(N=98)\end{array}$ & $\begin{array}{l}\text { Group P } \\
(N=98)\end{array}$ & $P$ value \\
\hline $\begin{array}{l}\text { Postoperative } \\
\text { complications }\end{array}$ & $40(40.8 \%)$ & $37(37.8 \%)$ & 0.661 \\
$\begin{array}{l}\text { Ileus } \\
\begin{array}{l}\text { Length of operation } \\
( \pm \mathrm{SD})\end{array}\end{array}$ & $617.1( \pm .1 \%)$ & $6(6.1 \%)$ & 1.000 \\
\begin{tabular}{l} 
Bleeding $( \pm \mathrm{SD})$ \\
\hline
\end{tabular} & $474.4( \pm 319.9)$ & $308.8( \pm 276.1)$ & $<0.001$ \\
\hline
\end{tabular}

Nevertheless, this study reports the surgical results of omentum-preserving gastrectomy for advanced gastric cancer by comparing well-balanced groups. These results may be worth confirming by means of a well-designed prospective study.

\section{Conclusion}

In this series, omentum-preserving gastrectomy for advanced gastric cancer did not increase the peritoneal relapse rate and did not affect the survival of patients compared to conventional gastrectomy. The non-inferiority of the omission of omentectomy should be evaluated in a randomized controlled trial.

Conflict of interest None declared.

\section{References}

1. Liebermann-Meffert D. The greater omentum. Anatomy, embryology, and surgical application. Surg Clin N Am 2000;80:275-293, xii

2. Platell C, Cooper D, Papadimitriou JM, et al. The omentum. World J Gastroenterol. 2000;6:169-76.

3. Higgins GM, Bain CG. The absorption and transference of particulate material by the great omentum. Surg Gynecol Obstet. 1930;50:851-60.

4. Hagiwara A, Takahashi T, Sawai K, Taniguchi H, Shimotsuma M, Okano S, et al. Milky-spots as the implantation site for malignant cells in the peritoneal dissemination in mice. Cancer Res. 1993;53:687-92.

5. Imada T, Matsumoto A. Mechanism by which peritoneal disseminated metastasis develops in gastric cancer. Nippon Geka Gakkai Zassi 1986;87(6):593-603 (in Japanese).

6. Lawrance RJ, Loizidou M, Cooper AJ, Alexandar P, Taylor I. Importance of the omentum in the development of intra-abdominal metastases. Br J Surg. 1991;78:117-9.

7. Hagiwara A, Sawai K, Sakakura C, Shirasu M, Ohgaki M, Yamasaki J, et al. Complete omentectomy and extensive lymphadenectomy with gastrectomy improves the survival of gastric cancer patients with metastases in the adjacent peritoneum. Hepatogastroenterology. 1998;45:1922-9.

8. Japanese Gastric Cancer Association. Japanese gastric cancer guidelines 2010 (ver. 3). Gastric Cancer. 2011;14:113-23.

9. Sakuramoto S, Sasako M, Yamaguchi T, Kinoshita T, Fujii M, Nashimoto A, et al. Adjuvant chemotherapy for gastric cancer with S-1, an oral fluoropyrimidine. N Engl J Med. 2007;357(18): 1810-20.

10. Fujita J, Tsukahara Y, Ikeda K, Akagi K, Suga K, Hata S, et al. Evaluation of omentum preserving gastrectomy for advanced gastric cancer. Jpn J Gastroenterol Surg 2003;36:1151-1158 (in Japanese).

11. Watanabe N, Nashimoto A, Yabusaki H, Takii Y, Tsuchiya K, Tanaka O. Evaluation of omento-bursectomy for T2 and T3 gastric cancers. Nippon Rinsho Geka Gakkai Zassi 2004;65(10): 2570-2574 (in Japanese).

12. Clavien PA, Barkun J, de Oliveira ML, Vauthey JN, Dindo D, Schulick RD, et al. The Clavien-Dindo classification of surgical complications: five-year experience. Ann Surg. 2009;250: 187-96.

13. Ha TK, An JY, Youn HG, Noh JH, Sohn TS, Kim S. Omentumpreserving gastrectomy for early gastric cancer. World J Surg. 2008;32:1703-8.

14. Fujita J, Kurokawa Y, Sugimoto T, Miyashiro I, Iijima S, Kimura Y, et al. Survival benefit of bursectomy in patients with resectable gastric cancer: interim analysis results of a randomized controlled trial. Gastric Cancer. 2012;15(1):42-8. 